\title{
Neoadjuvant capecitabine, radiotherapy, and bevacizumab (CRAB) in locally advanced rectal cancer: results of an open-label phase II study
}

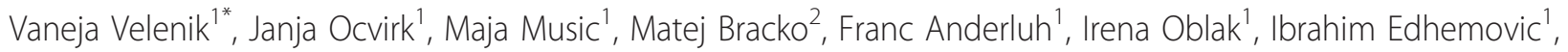
Erik Brecelj ${ }^{1}$, Mateja Kropivnik ${ }^{1}$ and Mirko Omejc ${ }^{2}$

\begin{abstract}
Background: Preoperative capecitabine-based chemoradiation is a standard treatment for locally advanced rectal cancer (LARC). Here, we explored the safety and efficacy of the addition of bevacizumab to capecitabine and concurrent radiotherapy for LARC.
\end{abstract}

Methods: Patients with MRI-confirmed stage II/III rectal cancer received bevacizumab $5 \mathrm{mg} / \mathrm{kg}$ i.v. 2 weeks prior to neoadjuvant chemoradiotherapy followed by bevacizumab $5 \mathrm{mg} / \mathrm{kg}$ on Days 1, 15 and 29, capecitabine $825 \mathrm{mg} /$ $\mathrm{m}^{2}$ twice daily on Days 1-38, and concurrent radiotherapy $50.4 \mathrm{~Gy}$ (1.8 Gy/day, 5 days/week for 5 weeks + three 1.8 Gy/day), starting on Day 1. Total mesorectal excision was scheduled 6-8 weeks after completion of chemoradiotherapy. Tumour regression grades (TRG) were evaluated on surgical specimens according to Dworak. The primary endpoint was pathological complete response ( $p C R)$.

Results: 61 patients were enrolled (median age 60 years [range 31-80], 64\% male). Twelve patients (19.7\%) had T3N0 tumours, 1 patient T2N1, 19 patients (31.1\%) T3N1, 2 patients (3.3\%) T2N2, 22 patients (36.1\%) T3N2 and 5 patients (8.2\%) T4N2. Median tumour distance from the anal verge was $6 \mathrm{~cm}$ (range 0-11). Grade 3 adverse events included dermatitis $(n=6,9.8 \%)$, proteinuria $(n=4,6.5 \%)$ and leucocytopenia $(n=3,4.9 \%)$. Radical resection was achieved in 57 patients (95\%), and 42 patients (70\%) underwent sphincter-preserving surgery. TRG 4 (pCR) was recorded in 8 patients (13.3\%) and TRG 3 in 9 patients (15.0\%). T-, N- and overall downstaging rates were 45.2\%, $73.8 \%$, and $73.8 \%$, respectively.

Conclusions: This study demonstrates the feasibility of preoperative chemoradiotherapy with bevacizumab and capecitabine. The observed adverse events of neoadjuvant treatment are comparable with those previously reported, but the $\mathrm{PCR}$ rate was lower.

Keywords: capecitabine, chemoradiation, bevacizumab, locally advanced rectal cancer, LARC, phase II study

\section{Introduction}

Treatment of locally advanced rectal cancer (LARC) is multimodal and generally consists of surgery, radiation and chemotherapy. Preoperative radiotherapy (RT) has been investigated as a neoadjuvant treatment for rectal cancer to improve local control and survival rates. The potential advantages of preoperative RT include decreased tumour spread (local and distant), reduced acute toxicity, increased sensitivity to RT and enhanced

\footnotetext{
* Correspondence: vvelenik@onko-i.si

'Institute of Oncology, Zaloska 2, 1000 Ljubljana, Slovenia

Full list of author information is available at the end of the article
}

sphincter preservation during surgery [1-4]. In LARC, the addition of 5-fluorouracil (5-FU) to preoperative RT has been shown to improve pathological complete response rate, tumour downstaging [5] and locoregional control [6,7] compared with RT alone. Furthermore, preoperative chemoradiotherapy improves locoregional control with less toxicity compared with postoperative chemoradiotherapy [4]. Thus, preoperative chemoradiotherapy with continuous infusional 5-FU has become a standard of care in rectal cancer, especially in tumours of the lower and middle rectum. 
The oral fluoropyrimidine capecitabine was designed to mimic continuous 5 -FU infusion and to generate 5 -FU preferentially in tumour tissue. Capecitabine has demonstrated efficacy comparable with intravenous 5-FU in metastatic colorectal cancer as well as in the adjuvant setting in colon cancers [8-14]. Furthermore, capecitabine has been investigated in various protocols for rectal and other gastrointestinal cancers in combination with RT [15]; indeed, equivalence of capecitabine plus RT and 5FU plus RT as preoperative therapy in LARC was demonstrated in the systematic review by Saif and colleagues [16]. Recently, two phase III trials, the large National Surgical Adjuvant Breast and Bowel Project (NSABP) R-04 Intergroup study [17] and a German trial [18], have confirmed that capecitabine is non-inferior to 5-FU as component of neoadjuvant radiochemotherapy in rectal cancer, and a retrospective analysis from a single centre found preoperative capecitabine plus RT to have more favourable results and higher downstaging rates that infusional 5-FU plus RT [19]. Preoperative capecitabine-based chemoradiation is now a standard treatment for LARC [4].

Phase II studies evaluating preoperative doublet chemotherapy of oxaliplatin or irinotecan plus 5-FU or capecitabine combined with concurrent radiotherapy in LARC have reported either no change or an increase in pathological complete response with the addition of oxaliplatin or irinotecan, and this addition also frequently resulted in increased acute toxicity $[17,18,20-26]$.

The addition of bevacizumab, a humanized monoclonal antibody to vascular endothelial growth factor (VEGF), to chemotherapy has been shown to increase the efficacy of therapy in metastatic colorectal cancer [27]. It is postulated that combining bevacizumab with chemoradiation may increase antitumour efficacy by maximizing inhibition of the VEGF pathway $[28,29]$. That said, there are relatively limited data on the safety and efficacy of bevacizumab in combination with chemotherapy and radiation in the neoadjuvant setting [30-34]. In this study we explored the safety and efficacy of neoadjuvant capecitabine, concurrent radiotherapy and bevacizumab (CRAB) in LARC.

\section{Patients and Methods}

We undertook a prospective, open-label, single-arm phase II study in patients with histologically proven adenocarcinoma of the rectum (Clinicaltrials.gov registration number: NCT00842686). The study was approved by the relevant institutional review board, the National Ethics Committee and the Ministry of Health. All patients gave written informed consent prior to any study procedure.

\section{Patients}

Patient pretreatment work-up comprised a complete history, physical examination, full blood count, serum biochemistry, carcinoembryonic antigen, chest radiography, ultrasonography and/or computed tomography (CT) scan of the whole abdomen. The extent of locoregional disease was determined by magnetic resonance imaging (MRI) of the pelvis of each patient. Eligible patients had to have a histologically verified stage II or III adenocarcinoma of the rectum, the disease had be considered either resectable at the time of entry or thought likely to become resectable after preoperative chemoradiation with no evidence of distant metastases. Other key inclusion criteria were: age 18-80 years; World Health Organization performance status of 0-2; adequate bone marrow, liver, renal and cardiac function (no history of clinically significant cardiovascular disease); no prior radiotherapy, chemotherapy or any targeting therapy for rectal cancer; ability to swallow oral medications; and signed informed consent. Key exclusion criteria included: other co-existing malignancy or malignancy within the last 5 years prior the enrolment other than non-melanoma skin cancer or in situ carcinoma of the cervix; patients with severe concurrent medical or psychiatric illness; a known hypersensitivity to study drug; and pregnant or lactating patients.

\section{Study design and treatment}

The study design and treatment schedule are shown in Figure 1. Three-dimensional CT-based treatment planning was performed. The CT was taken on treatment position with $5 \mathrm{~mm}$ thick slices. The clinical target volume was defined as covering the small pelvis from the L5-S1 interspace to $5 \mathrm{~cm}$ below the primary tumour. The lateral borders were $5 \mathrm{~mm}$ outside the true bony pelvis. The posterior margin covered the sacrum and the anterior margin encompassed the posterior onethird to one-half of the bladder and/or vagina. An additional $1 \mathrm{~cm}$ in all directions was added to the clinical target volume to obtain the planning target volume. The dose was prescribed to cover the planning target volume with a $95 \%$ reference isodose $(95 \%$ of the International Commission on Radiation Unit point dose).

RT was initiated on Day 1. Patients received a total irradiation dose of 45 Gy to the pelvis plus 5.4 Gy as a boost to the primary tumour in 1.8 Gy daily fractions over 5.5 weeks. Radiotherapy was delivered using 15 MV photon beams and four-field box technique, once daily, 5 days per week. All fields were treated daily. Multileaf collimators were used to shape individual radiation fields and for the protection of normal tissues. Patients were irradiated in a prone position with a full bladder and using a belly board to minimize exposure of the small bowel.

Chemotherapy was administered concomitantly with RT and consisted of oral capecitabine at a daily dose of $1650 \mathrm{mg} / \mathrm{m}^{2}$, divided into two equal doses given 12 


\section{Histologically proven stage II/III rectal cancer}

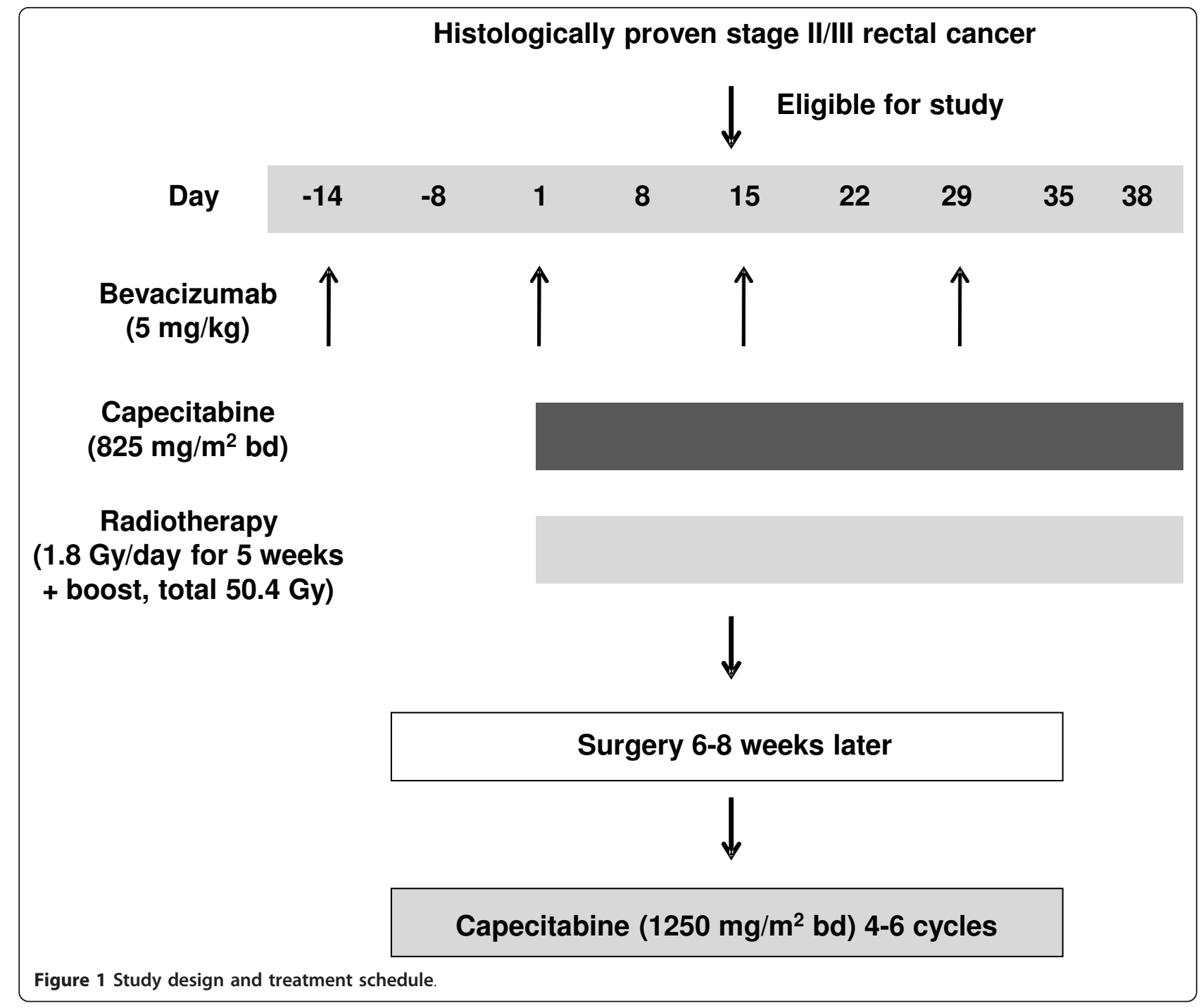

hours apart. One dose was taken 1 hour prior to RT. The chemotherapy started on the first day of RT (Day 1 ), finished on the last day of RT (Day 38) and was continuous throughout the RT period (i.e. it included weekends). Bevacizumab was administered at a dose of $5 \mathrm{mg} /$ $\mathrm{kg}$ on treatment days: $-14,1,15$ and 29 . The drug was delivered as an intravenous infusion over a 30-90-min period.

Resection was performed 6-8 weeks after the completion of chemoradiotherapy. A total mesorectal excision was the recommended operation for mid and distal rectal tumours. Surgical management included a sphincterpreservation approach whenever possible. The option for a temporary colostomy during surgery was left to the surgeon's discretion. Complications after surgery were recorded.

In patients achieving histopathological R0 or R1 resection, adjuvant chemotherapy was recommended: this comprised capecitabine $1250 \mathrm{mg} / \mathrm{m}^{2}$ orally twice daily on Days 1-14 every 3 weeks; 4 (R0 resection) or 6 cycles (R1 resection) were recommended, beginning 6-8 weeks after surgery.

\section{Study assessments}

It has been shown that complete eradication of the primary tumour observed in the histopathological specimen (pathological complete response [pCR]) correlates with a favourable overall prognosis, so obtaining a pCR might be beneficial [35]. Thus, the primary endpoint of pCR rate was selected for the current analysis. Secondary endpoints were: pathological response rate (plus tumour regression grade [TRG] according to Dworak scale); rate of sphincter-sparing surgical procedure; histopathological R0 resection rate; acute and late toxicity (SOMA/LENT scale); locoregional failure rate; diseasefree survival; and overall survival. The effect of 
preoperative chemoradiotherapy on tumour downstaging was assessed by comparing the pretreatment radiologically determined TNM stage with the postoperative pathologic TNM stage. As an exploratory objective, the mutation status of KRAS in pre-therapeutic biopsies was established and correlation to pathological response was assessed.

During treatment, patients were evaluated weekly. Clinical examinations, complete blood count and serum chemistry analysis were performed. Adverse events were assessed according to National Cancer Institute Common Toxicity Criteria (NCI-CTC) version 3.0. Re-evaluation of the primary tumour with pelvic MRI was performed four weeks after the completion of preoperative treatment.

Postoperative, pathological evaluation of the surgical specimen was performed. $\mathrm{pCR}$ was defined as the complete disappearance of all tumour cells. Histological regression of the primary tumour was semi-quantitatively determined according to a 5-point TRG scale: TRG 0, no regression; TRG 1, minimal regression (dominant tumour mass with obvious fibrosis and/or vasculopathy); TRG 2, moderate regression (predominantly fibrotic changes with few tumour cells or groups); TRG 3, good regression (very few tumour cells in fibrotic tissue); TRG 4, total regression (no tumour cells, only fibrotic mass).

Follow-up visits were scheduled at 3, 6, 12, 18, 24, 36, 48 and 60 months following the end of adjuvant chemotherapy.

\section{Statistical analysis}

The primary endpoint of the study was pCR rate. In the medical literature, phase II studies of capecitabine and RT suggest a pCR rate in the range of 4-31\%, while in our published study the pCR rate was approximately $9 \%$. We aimed to evaluate whether a $23 \%$ pCR rate could be achieved by adding bevacizumab to standard preoperative treatment. Setting $10 \%$ as the lowest $\mathrm{pCR}$ rate of interest, and with alpha error of $5 \%$ and power of $80 \%$, at least 50 evaluable patients were needed (calculated using power sample calculation, for two samples, percentages, $\alpha=5 \%, 1-\beta=20 \%$ ). Assuming that $\geq 10 \%$ of patients would not be evaluable, the estimated sample size required was at least 60 patients.

Statistics were descriptive and all data were analysed using the SPSS statistical software package, version 13 (SPSS Inc., Chicago, IL, USA).

\section{Results}

\section{Patient characteristics and treatment rates}

Between February 2009 and March 2010, a total of 61 patients were recruited. Patients' baseline and disease characteristics are summarized in Table 1. Three
Table 1 Patients' baseline and disease characteristics

\begin{tabular}{lc}
\hline Characteristics & $\begin{array}{c}\text { Patients } \\
(\mathbf{n}=61)\end{array}$ \\
\hline Median age, years (range) & $60(31-80)$ \\
Gender, $\mathrm{n}(\%)$ : & \\
$\quad$ Male & $39(64)$ \\
$\quad$ Female & $22(36)$ \\
WHO performance status, $\mathrm{n}(\%)$ & \\
0 & $52(85)$ \\
1 & $9(15)$ \\
TN clinical stage, $\mathrm{n}(\%)$ & \\
$\quad$ T3N0 & $12(19.7)$ \\
$\quad$ T2N1 & $1(1.7)$ \\
$\quad$ T3N1 & $19(31.1)$ \\
$\quad$ T2N2 & $2(3.3)$ \\
T3N2 & $22(36.1)$ \\
$\quad$ T4N2 & $5(8.2)$ \\
Median clinical tumour size per MRI, cm (range) & $6(1-12)$ \\
Median tumour distance from anal verge, cm (range) & $6(0-11)$ \\
Type of surgery ${ }^{a}, \mathrm{n}$ (\%) & \\
Low anterior resection & $35(57.4)$ \\
Coloanal reconstruction & $10(16.4)$ \\
Abdominoperineal resection & $14(23.0)$ \\
Pelvic exenteration & $2(3.3)$ \\
\hline
\end{tabular}

${ }^{a}$ As planned before the start of preoperative chemoradiotherapy.

MRI, magnetic nuclear imaging; $\mathrm{N}$, node; $\mathrm{T}$, tumour; WHO, World Health Organization.

patients $(4.9 \%)$ presented with stage T2 disease, 53 (86.9\%) with stage T3, 5 (8.2\%) with stage T4; lymph node involvement was detected in 49 patients $(80.3 \%)$. The most frequent MRI staging was uT3N+ (67\%). In 28 patients $(45.9 \%)$ the tumour invaded the mesorectal fascia and in half of the patients $(50 \%)$ the primary tumour was sited $\leq 5 \mathrm{~cm}$ from the anal verge.

All patients received neoadjuvant chemoradiotherapy plus bevacizumab. Treatment was terminated in one patient as a result of withdrawal of informed consent following four weeks of treatment. All other patients received $100 \%$ of the expected radiation treatment. Treatment interruption was necessary for 7 patients (11.6\%) because of grade $2(n=2)$ and grade $3(n=3)$ leucopenia, grade 3 diarrhoea $(\mathrm{n}=1)$, and grade $3(\mathrm{n}=$ $1)$ and grade $4(n=1)$ vascular toxicity. Other grade 3 toxicities included dermatitis $(n=6)$, proteinuria $(n=4)$ and hypertension $(n=1)$. There were no treatmentrelated deaths during the study.

RT was interrupted for 2-7 days as a result of treatment (median interruption: 2 days), while 56 patients (91\%) received $95-100 \%$ of the designated chemotherapy dose. Overall, 58 patients $(95.1 \%)$ received all four infusions of bevacizumab while the remaining 3 patients received three infusions. 


\section{Treatment-related toxicities}

The frequency and grade of treatment-related acute toxicities are summarised in Table 2. The most frequent adverse event reported with chemoradiotherapy was grade 2 and 3 radiodermatitis. During treatment, 25 patients lost weight; the maximum body weight loss was $6.5 \%$ (median 3.3\%). Of the remaining patients, 26 maintained a constant weight and nine experienced a weight increase of up to $5 \%$ (median: $2.4 \%$ ).

\section{Surgery rates and outcomes}

All patients underwent definitive surgery, although one patient revealed distant metastases after completion of chemoradiotherapy. Surgery was performed 25 to 79 days (median: 55 days) after the last day of chemoradiotherapy. Exploratory surgery was performed in only 1 patient because of a large, unresectable T4 tumour with peritoneal carcinomatosis. The median hospital stay for surgery was 11 days (range: 7-32 days).

Radical resection was achieved in 57 patients (95\%) and 42 patients $(70 \%)$ underwent sphincter-preserving surgery. A temporary stoma was created in 41 patients. In one patient pathohistological examination of the surgical specimen revealed malignant melanoma; this patient was considered misdiagnosed and excluded from the efficacy analysis.

Pathological TNM stages in relation to preoperative TNM status are presented in Table 3. TRG 4 (pCR) was recorded in $8 / 60$ patients (13.3\%) and TRG 3 in 9/60

Table 2 Acute toxicities occurring during preoperative chemoradiotherapy

\begin{tabular}{|c|c|c|c|c|}
\hline \multirow[b]{2}{*}{ Toxicity } & \multicolumn{4}{|c|}{ Patients, n (\%) } \\
\hline & Grade 1 & Grade 2 & Grade 3 & Grade 4 \\
\hline \multicolumn{5}{|l|}{ Haematological: } \\
\hline Leucocytopenia & $12(19.7)$ & $5(8.2)$ & $3(4.9)$ & - \\
\hline Anaemia & $5(8.2)$ & - & - & - \\
\hline \multicolumn{5}{|l|}{ Non-haematological: } \\
\hline Diarrhoea & $14(22.9)$ & $4(6.5)$ & $1(1.6)$ & - \\
\hline Fatigue & $7(11.5)$ & $3(4.9)$ & - & - \\
\hline Nausea & $5(8.2)$ & - & - & - \\
\hline Anorexia & $2(3.3)$ & - & - & - \\
\hline Dermatitis & $3(4.9)$ & $14(22.9)$ & $6(9.8)$ & - \\
\hline Hand-food syndrome & $5(8.2)$ & $2(3.3)$ & - & - \\
\hline Cystitis & $3(4.9)$ & - & - & - \\
\hline Hepatotoxicity & $2(3.3)$ & $2(3.3)$ & - & - \\
\hline Vascular & - & - & $1(1.6)$ & $1(1.6)$ \\
\hline Proteinuria & $10(16.4)$ & $2(3.3)$ & $4(6.5)$ & - \\
\hline Hypertension & $2(3.3)$ & $2(3.3)$ & $1(1.6)$ & - \\
\hline Infection & $3(4.9)$ & $5(8.2)$ & - & - \\
\hline Pain & $20(32.8)$ & $3(4.9)$ & - & - \\
\hline Bleeding & $10(16.4)$ & - & - & - \\
\hline
\end{tabular}

According to National Cancer Institute Common Toxicity Criteria (version 3) patients (15.0\%). T-, $\mathrm{N}$ - and overall downstaging rates were $46.7 \%, 65.0 \%$ and $75.0 \%$, respectively.

KRAS mutations were found in 20 (33.9\%) out of 59 bioptic tumour samples obtained before preoperative treatment. KRAS status was not associated with pathological response.

In total, 38 patients (62.3\%) developed perioperative complications. The most frequent were delayed wound healing ( $\mathrm{n}=18,30.0 \%)$, infection/abscess $(\mathrm{n}=12$, $20.0 \%)$ and anastomotic leakage $(n=7,11.7 \%)$. Six patients required surgical re-intervention for anastomotic leakage $(n=3)$, abdominal abscess $(n=2)$ and pneumothorax $(n=1)$. There were no perioperative deaths. A summary of perioperative toxicity is shown in Table 4.

Postoperative chemotherapy was administered to 51 (83.6\%) patients. Reasons for not administering adjuvant chemotherapy were: progression of the disease $(n=2)$, misdiagnosis $(n=1)$; withdrawal from study $(n=1) ;>8$ week interval between the operation and adjuvant therapy $(n=1)$; and postoperative complications $(n=5)$. Postoperative chemotherapy comprised capecitabine $1250 \mathrm{mg} / \mathrm{m}^{2}$ on Days 1-14 every 3 weeks for 4 or 6 cycles. A total of 42 patients received all planned cycles. Two patients only received 3 cycles because of disease progression $(n=1)$ and death because of pulmonary thromboembolism $(\mathrm{n}=1) ; 2$ patients only received 2 cycles because of diarrhoea and dehydration $(n=1)$ and nonspecific chest pain $(\mathrm{n}=1)$; and 3 patients only received 1 cycle because of the development of presacral abscesses $(n=2)$ and nonspecific chest pain $(n=1)$.

\section{Discussion}

This phase II study demonstrates the feasibility of preoperative chemoradiation with bevacizumab and capecitabine in patients with LARC. Indeed, a high R0 resection rate was achieved despite tumour invasion of the mesorectal fascia in $46 \%$ of patients. A well-accepted approach in the management of LARC is neoadjuvant fluoropyrimidine-based chemoradiation and a number of prospective and retrospective trials have suggested that preoperative capecitabine is at least equivalent to infusional 5-fluorouracil when combined with RT [16-19], and may improve tumour downstaging. In 2009, the US National Comprehensive Cancer Network recommended capecitabine as an acceptable alternative to 5-FU in this setting [36].

The pCR rate of $13 \%$ observed with neoadjuvant capecitabine plus bevacizumab plus RT was similar to an earlier phase II study by our group examining neoadjuvant single-agent capecitabine plus RT in LARC [37]. This $\mathrm{pCR}$ rate, albeit relatively low, is within the range $0-31 \%$ reported across a number of phase II studies evaluating single-agent capecitabine plus RT [38-43]. In 
Table 3 Distribution of postoperative pathological TMN stages compared with pretreatment clinical stages $(\mathrm{n}=60$ )

\begin{tabular}{|c|c|c|c|c|c|c|c|c|}
\hline \multirow[t]{2}{*}{ Before } & \multicolumn{8}{|c|}{ After surgery (pTNM) } \\
\hline & TONO & T1No & T2NO & T3NO & T2N1 & T3N1 & T4N1 & T3N2 \\
\hline T3NO & 3 & & 4 & 3 & & 2 & & \\
\hline T2N1 & & & 1 & & & & & \\
\hline T3N1 & 5 & 3 & 4 & 4 & 1 & 1 & & \\
\hline $\mathrm{T} 2 \mathrm{~N} 2$ & & & 1 & & & & & 1 \\
\hline T3N2 & & 1 & 2 & 13 & & 1 & 1 & 4 \\
\hline T4N2 & & 1 & 2 & 1 & & 1 & & \\
\hline Total & $8(13.3 \%)$ & $5(8.3 \%)$ & $14(23.3 \%)$ & $21(35 \%)$ & $1(1.7 \%)$ & $5(8.3 \%)$ & $1(1.7 \%)$ & $5(8.3 \%)$ \\
\hline
\end{tabular}

c - Clinical, p - pathological, T - Tumour, N - Node, M - Metastasis.

one of the largest studies performed to date, the pCR rate was $12 \%$ [44], and in the recently presented NSABP-04 study the pCR ranged from 18 to $22 \%$ with capecitabine and 5-FU achieving similar rates of improvement but no additional benefit being observed with the addition of oxaliplatin to either of these agents [17]. A study by Ofner and co-workers [45] evaluating preoperative capecitabine and oxaliplatin reported a pCR rate of $10 \%$ while studies investigating preoperative capecitabine, oxaliplatin and bevacizumab found rates of $24-36 \%$ [32-34].

In the phase II trial by Crane and coworkers [31], 25 patients with LARC received neoadjuvant chemoradiotherapy with bevacizumab (three doses of $5 \mathrm{mg} / \mathrm{kg}$ given every 2 weeks) and capecitabine $\left(900 \mathrm{mg} / \mathrm{m}^{2}\right.$ orally twice daily on days of radiation therapy), followed by surgical resection a median of 7.3 weeks later. These authors reported a pCR rate of $32 \%$ (8 patients), which is considerably higher than that reported here. One possible explanation for the relatively low $\mathrm{pCR}$ rate observed in our study was that the patients in this study had advanced tumours; indeed, most of the patients had regionally advanced disease and in almost half of the patients the tumour had invaded the mesorectal fascia. However, caution is needed when comparing $\mathrm{pCR}$ rates as the pCR rate itself is highly dependent on the quality of the pathological examination [46] and a longer interval between end of chemoradiotherapy and surgery (6-8 weeks vs. 2 weeks) has been reported to increase pCR rate without reducing local recurrence rate or survival $[47,48]$. While there has been much debate about whether $\mathrm{pCR}$ is associated with a favourable long-term

Table 4 Perioperative adverse events $(n=60)$

\begin{tabular}{lc}
\hline Complication & Patients, $\mathbf{n}(\%)^{\mathbf{a}}$ \\
\hline Delayed healing of postoperative wound & $18(30.0)$ \\
Infection/abscess & $12(20.0)$ \\
Pneumothorax & $1(1.7)$ \\
Anastomotic leakage & $7(11.7)$ \\
\hline
\end{tabular}

a Patients could have more than one adverse event. outcome, a recently published pooled analysis of data from 3105 patients from 14 studies would suggest that patients with $\mathrm{pCR}$ after chemoradiation have better long-term outcome than those without pCR [49].

The adverse event profile observed during neoadjuvant capecitabine plus bevacizumab chemoradiotherapy was comparable with those reported in an earlier study involving capecitabine plus bevacizumab with concurrent RT [31]. The most frequent preoperative adverse events were dermatitis, pain and leucopenia, and adverse events related to bevacizumab therapy were relatively infrequent. Any postoperative adverse events were mainly related to delayed wound healing and infection/ abscess. No treatment-related deaths were recorded. These results, together with those of Crane and coworkers [31] suggest that the combination of neoadjuvant capecitabine plus bevacizumab with concurrent RT is feasible and well tolerated in the treatment of LARC. A high radical resection rate suggests its potential positive effect on tumour downstaging. The observed adverse events during neoadjuvant treatment in our study are comparable with those reported previously; however, no clinically relevant increase in pathologic response rate was observed. Longer follow-up is needed to assess the impact on other efficacy endpoints.

Long-term follow-up data on survival and local control in patients with LARC having undergone neoadjuvant capecitabine plus bevacizumab chemoradiotherapy followed by surgery are eagerly awaited. It will also be interesting to compare any long-term follow-up data with that which is currently available at the moment on single-agent capecitabine-based chemoradiotherapy $[39,50]$ to help determine the benefits of adding bevacizumab to the regimen.

\section{Conclusion}

The results of this phase II study indicate that neoadjuvant capecitabine chemoradiotherapy is an effective treatment for patients with LARC and the incorporation of bevacizumab into a standard capecitabine-based chemoradiotherapy regimen is feasible and well tolerated. 


\section{List of abbreviations}

5-FU: 5-fluorouracil; CT: computed tomography; LARC: locally advanced rectal cancer; MRI: magnetic resonance imaging; pCR: pathological complete response; RT: radiotherapy; TRG: tumour regression grade; VEGF: vascular endothelial growth factor.

\section{Acknowledgements}

This was an investigator-initiated trial funded by Roche.

\section{Author details}

${ }^{1}$ Institute of Oncology, Zaloska 2, 1000 Ljubljana, Slovenia. ${ }^{2}$ University Medical Centre, Zaloska 7, 1000 Ljubljana, Slovenia.

\section{Authors' contributions}

W: contributions to conception and design, acquisition of data, analysis and interpretation of data; involvement in drafting and reviewing the manuscript. JO: contribution to acquisition of data, analysis and interpretation of data. MM: contribution to acquisition of data, analysis and interpretation of data. MB: contribution to acquisition of data. FA: contribution to acquisition of data, analysis and interpretation of data. IO: contribution to acquisition of data.

IE: contribution to acquisition of data, analysis and interpretation of data. EB: contribution to acquisition of data, analysis and interpretation of data. MK: contribution to acquisition of data. MO: contributions to acquisition of data, analysis and interpretation of data; involvement in drafting and reviewing the manuscript. All authors have read and approved the final version of the manuscript.

\section{Competing interests}

This was an investigator-initiated trial supported by Roche. The authors declare that they have no financial or non-financial competing interests.

Received: 19 June 2011 Accepted: 31 August 2011

Published: 31 August 2011

\section{References}

1. Camma C, Giunta M, Fiorica F, Pagliaro L, Craxi A, et al: Preoperative radiotherapy for resectable rectal cancer: a meta-analysis. JAMA 2000, 284:1008-1015.

2. Glimelius B, Isacsson U, Jung B, Pahlman L: Radiotherapy in addition to radical surgery in rectal cancer: evidence for a dose-response effect favoring preoperative treatment. Int I Radiat Oncol Biol Phys 1997, 37:281-287.

3. Pahlman L, Glimelius B: Pre- or postoperative radiotherapy in rectal and rectosigmoid carcinoma. Report from a randomized multicentre trial. Ann Surg 1990, 211:187-195.

4. Sauer R, Becker H, Hohenberger W, Rödel C, Wittekind C, German Rectal Cancer Study Group, et al: Preoperative versus postoperative chemoradiotherapy for rectal cancer. N Engl J Med 2004, 351:1731-1740.

5. Bosset JF, Calais G, Mineur L, Maingon P, Radosevic-Jelic L, et al: Enhanced tumorocidal effect of chemotherapy with preoperative radiotherapy for rectal cancer: preliminary results - EORTC 22921. J Clin Oncol 2005, 23:5620-5627.

6. Bosset JF, Collette L, Calais G, Mineur L, Maingon P, EORTC Radiotherapy Group Trial 22921, et al: Chemotherapy with preoperative radiotherapy in rectal cancer. N Engl J Med 2006, 355:1114-1123.

7. Gérard JP, Conroy T, Bonnetain F, Bouché O, Chapet O, et al: Preoperative radiotherapy with or without concurrent fluorouracil and leucovorin in T3-4 rectal cancers: results of FFCD 9203. J Clin Oncol 2006, 24:4620-4625.

8. Bajetta E, Beretta E, Di Bartolomeo M, Mariani L, Valvo F, et al: Capecitabine chemoradiation for rectal cancer after curative surgery. $J$ Chemother 2006, 18:85-89.

9. Cassidy J, Twelves C, Van Cutsem E, Hoff P, Bajetta E, Capecitabine Colorectal Cancer Study Group, et al: First-line oral capecitabine therapy in metastatic colorectal cancer: a favorable safety profile compared with intravenous 5-fluorouracil/leucovorin. Ann Oncol 2002, 13:566-575.

10. Hoff PM, Ansari R, Batist G, Cox J, Kocha W, et al: Comparison of oral capecitabine versus intravenous fluorouracil plus leucovorin as first-line treatment in 605 patients with metastatic colorectal cancer: results of a randomised phase III study. J Clin Oncol 2001, 19:2282-2292.
11. Twelves C, Wong A, Nowacki MP, Abt M, Burris H, et al: Capecitabine as adjuvant treatment for stage III colon cancer. N Engl J Med 2005, 352:2696-2704.

12. Van Cutsem E, Findlay M, Osterwalder B, Kocha W, Dalley D, et al: Capecitabine, an oral fluoropyrimidine carbamate with substantial activity in advanced colorectal cancer: results of a randomized phase II study. J Clin Oncol 2000, 18:1337-1345.

13. Van Cutsem E, Twelves C, Cassidy J, Allman D, Bajetta E, et al: Oral capecitabine compared with intravenous fluorouracil plus leucovorin in patients with metastatic colorectal cancer: results of a large phase III study. J Clin Oncol 2001, 19:4097-4106.

14. Van Cutsem E, Hoff PM, Harper P, Bukowski RM, Cunningham D, et al: Oral capecitabine vs intravenous 5 -fluorouracil and leucovorin: integrated efficacy data and novel analyses from two large, randomized, phase III trials. Br J Cancer 2004, 90:1190-1197.

15. Glynne-Jones R, Dunst J, Sebag-Montefiore D: The integration of oral capecitabine into chemoradiation regimens for locally advanced rectal cancer: how successful have we been? Ann Oncol 2006, 17:361-371.

16. Saif MW, Hashmi S, Zelterman D, Almhanna K, Kim R: Capecitabine vs continuous infusion 5-FU in neoadjuvant treatment of rectal cancer. A retrospective review. Int J Colorectal Dis 2008, 23:139-145.

17. Roh MS, Yothers GA, O'Connell MJ, Beart RW, Pitot HC, et al: The impact of capecitabine and oxaliplatin in the preoperative multimodality treatment in patients with carcinoma of the rectum: NSABP R-04. J Clin Oncol 2011, 29(suppl):Abst 3503..

18. Hofheinz R, Wenz FK, Post S, Matzdorff A, Laechelt S, et al: Capecitabine (Cape) versus 5-fluorouracil (5-FU)-based (neo)adjuvant chemoradiotherapy (CRT) for locally advanced rectal cancer (LARC): Long-term results of a randomized, phase III trial. J Clin Oncol 2011, 29(suppl):Abst 3504.

19. Kim DY, Jung KH, Kim TH, Kim DW, Chang HJ, et al: Comparison of 5fluorouracil/leucovorin and capecitabine in preoperative chemoradiotherapy for locally advanced rectal cancer. Int I Radiat Oncol Biol Phys 2007, 67:378-384.

20. Gérard JP, Azria D, Gourgou-Bourgade S, Martel-Laffay I, Hennequin C, et al: Comparison of two neoadjuvant chemoradiotherapy regimens for locally advanced rectal cancer: results of the phase III trial ACCORD 12/ 0405-Prodige 2. J Clin Oncol 2010, 28:1638-1644.

21. Aschele C, Cionini L, Lonardi S, Pinto C, Cordio S, et al: Primary tumor response to preoperative chemoradiation with or without oxaliplatin in locally advanced rectal cancer: Pathologic results of the STAR-01 randomized phase III trial. J Clin Oncol 2011, 29:2773-2780.

22. Rödel $\mathrm{C}$, Becker H, Fietkau R, Graeven U, Hohenberger W, et al: Preoperative chemoradiotherapy and postoperative chemotherapy with 5 -fluorouracil and oxaliplatin versus 5 -fluorouracil alone in locally advanced rectal cancer: first results of the German CAO/ARO/AIO-04 randomized phase III trial. J Clin Oncol 2011, 29(suppl):Abst LBA3505.

23. Mohiuddin M, Winter K, Mitchell E, Hanna N, Yuen A, et al: Randomized phase II study of neoadjuvant combined-modality chemoradiotherapy for distal rectal cancer: radiation therapy oncology group trial 0012. J Clin Oncol 2006, 24:650-655.

24. Klautke G, Kuchenmeister U, Foitzik T, Ludwig K, Prall F, et al: Concurrent chemoradiotherapy with capecitabine and weekly irinotecan as preoperative treatment for rectal cancer: results from a phase I/II study. Br J Cancer 2006, 94:976-981.

25. Navarro M, Dotor E, Rivera F, Sánchez-Rovira P, Vega-Villegas ME, et al: A phase II study of preoperative radiotherapy and concomitant weekly irinotecan in combination with protracted venous infusion 5 -fluorouracil, for resectable locally advanced rectal cancer. Int I Radiat Oncol Biol Phys 2006, 66:201-205.

26. Willeke F, Horisberger K, Kraus-Tiefenbacher U, Wenz F, Leitner A, et al: A phase II study of capecitabine and irinotecan in combination with concurrent pelvic radiotherapy (Caplri-RT) as neoadjuvant treatment of locally advanced rectal cancer. Br J Cancer 2007, 96:912-917.

27. Giantonio BJ, Catalano PJ, Meropol NJ, O'Dwyer PJ, Mitchell EP, Eastern Cooperative Oncology Group Study E3200, et al: Bevacizumab in combination with oxaliplatin, fluorouracil, and leucovorin (FOLFOX4) for previously treated metastatic colorectal cancer: results from the Eastern Cooperative Oncology Group Study E3200. J Clin Oncol 2007, 25:1539-1544. 
28. Willett CG, Boucher Y, di Tomaso E, Duda DG, Munn LL, et al: Direct evidence that the VEGF-specific antibody bevacizumab has antivascular effects in human rectal cancer. Nat Med 2004, 10:145-147.

29. Willett CG, Boucher $Y$, Duda DG, di Tomaso E, Munn LL, et al: Surrogate markers for antiangiogenic therapy and dose-limiting toxicities for bevacizumab with radiotherapy and chemotherapy: continued experience of a phase I trial in rectal cancer patients. J Clin Oncol 2005, 23:8136-8139.

30. Czito BG, Bendell JC, Willet CG, Morse MA, Blobe GC, et al: Bevacizumab, oxaliplatin, and capecitabine with radiation therapy in rectal cancer: Phase I trial results. Int J Radiat Oncol Biol Phys 2007, 68:472-478.

31. Crane CH, Eng C, Feig BW, Das P, Skibber JM, et al: Phase II trial of neoadjuvant bevacizumab, capecitabine, and radiotherapy for locally advanced rectal cancer. Int J Radiat Oncol Biol Phys 2010, 76:824-830.

32. Resch G, De Vries A, Ofner D, Eisterer W, Rabl H, et al: Preoperative treatment with capecitabine, bevacizumab and radiotherapy for locally advanced rectal cancer - A two stage phase II trial. Radiother Oncol 2011.

33. Kennecke H, Berry S, Wong R, Zhou C, Tankel K, et al: Pre-operative bevacizumab, capecitabine, oxaliplatin and radiation among patients with locally advanced or low rectal cancer: A phase II trial. Eur J Cancer 2011.

34. Nogue M, Salud A, Vincente P, Arrivi A, Roca JM, et al: Addition of bevacizumab to XELOX induction therapy plus concomitant capecitabine-based chemoradiotherapy in magnetic resonance imagingdefined poor-prognosis locally advanced rectal cancer: the AVACROSS study. Oncologist 2011, 16:614-620.

35. Rodel C, Martus P, Papadoupolos T, Füzesi L, Klimpfinger M, et al: Prognostic significance of tumor regression after preoperative chemoradiotherapy for rectal cancer. J Clin Oncol 2005, 23:8688-8696.

36. National Comprehensive Cancer Network: National Comprehensive Cancer Network clinical practice guidelines, Rectal cancer.[http://www.nccn.org/ professionals/physician_gls/f_guidelines.asp\#site].

37. Velenik V, Anderlih F, Oblak I, Strojan P, Zakotnik B: Capecitabine as a radiosensitizing agent in neoadjuvant treatment of locally advanced respectable rectal cancer: prospective phase II trial. Croat Med J 2006, 47:693-700.

38. Craven I, Crellin A, Cooper R, Melcher A, Byrne P, et al: Preoperative radiotherapy combined with 5 days per week capecitabine chemotherapy in locally advanced rectal cancer. Br J Cancer 2007, 97:1333-1337.

39. Dunst J, Debus J, Rudat V, Wulf J, Budach W, et al: Neoadjuvant capecitabine combined with standard radiotherapy in patients with locally advanced rectal cancer. Strahlenther Onkol 2008, 184:450-456.

40. De Paoli A, Chiara S, Luppi G, Friso ML, Beretta GD, et al: Capecitabine in combination with preoperative radiation therapy in locally advanced, resectable rectal cancer: a multicentric phase II study. Ann Oncol 2006, 17:246-251.

41. Desai SP, El-Rayes BF, Ben-Josef E, Greenson JK, Knol JA, et al: A phase II study of preoperative capecitabine and radiation therapy in patients with rectal cancer. Am J Clin Oncol 2007, 30:340-345.

42. Dupuis $\mathrm{O}$, Vie B, Liedo G, Hennequin C, Noirclerc M, et al: Preoperative treatment combining capecitabine with radiation therapy in rectal cancer: a GERCOR phase II study. Oncology 2007, 73:169-176.

43. Lay GC, Caraul B, Dessi M, Orrù S, Murtas R, et al: Phase II study of preoperative irradiation and chemotherapy with capecitabine in patients with locally advanced rectal carcinoma. J Exp Clin Cancer Res 2007, 26:61-70.

44. Kim JC, Kim TW, Kim JH, Yu CS, Kim HC, et al: Preoperative concurrent radiotherapy with capecitabine before total mesorectal excision in locally advanced rectal cancer. Int J Radiat Oncol Biol Phys 2005, 63:346-353.

45. Ofner D, De Vries AF, Schabert-Moser R, Greil R, Rabl H, et al: Preoperative oxaliplatin, capecitabine, and external beam radiotherapy in patients with newly diagnosed, primary operable, cT\#NxM0, low rectal cancer: a phase II study. Strahlenther Onkol 2011, 187:100-107.

46. Chua YJ: Pathological complete response: still a relevant endpoint in rectal cancer? Lancet Oncol 2010, 11:807-808.

47. Francois Y, Nemoz CJ, Baulieux J, Vignal J, Grandjean JP, et al: Influence of the interval between preoperative radiation therapy and surgery on downstaging and the rate of sphincter-saving surgery for rectal cancer: the Lyon R90-01 randomized trial. J Clin Oncol 1999, 17:2396.
48. Glehen O, Chapet O, Adham M, Nemoz JC, Gerard JP: Long-term results of the Lyons R90-01 randomized trial of preoperative radiotherapy with delayed surgery and its effect on sphincter-saving surgery in rectal cancer. Br J Surg 2003, 90:996-998.

49. Maas M, Nelemans PJ, Valentini V, Das P, Rödel C, et al: Long-term outcome in patients with a pathological complete response after chemoradiation for rectal cancer: a pooled analysis of individual patient data. Lancet Oncol 2010, 11:835-844.

50. Velenik V, Oblak I, Anderluh F: Long-term results from a randomized phase II trial of neoadjuvant combined-modality therapy for locally advanced rectal cancer. Radiat Oncol 2010, 5:88.

doi:10.1186/1748-717X-6-105

Cite this article as: Velenik et al:: Neoadjuvant capecitabine, radiotherapy, and bevacizumab (CRAB) in locally advanced rectal cancer: results of an open-label phase II study. Radiation Oncology 201 6:105.

\section{Submit your next manuscript to BioMed Central and take full advantage of:}

- Convenient online submission

- Thorough peer review

- No space constraints or color figure charges

- Immediate publication on acceptance

- Inclusion in PubMed, CAS, Scopus and Google Scholar

- Research which is freely available for redistribution

Submit your manuscript at www.biomedcentral.com/submit
Ciomed Central 